\title{
De la construcción entramada a la introducción de estructuras metálicas en Madrid
}

\author{
From timber-framed houses to the introduction of steel structures in Madrid \\ E. González-Redondo ${ }^{*}$
}

\section{RESUMEN}

El análisis de edificios construidos con entramado de madera que se han conservado y el estudio de los contratos de obra originales, ha permitido comparar estas construcciones. Se fundamenta en tres pilares básicos: la investigación y el análisis de contratos de obra originales desde 1669 hasta 1900; el análisis gráfico de este tipo de edificaciones, algunas desaparecidas y otras que han conservado; y la experiencia constructiva que ofrece la inspección de numerosos edificios con daños observables. Esta investigación se apoya en el recorrido detallado de una esquina singular con más de tres siglos de historia. Se puede considerar como edificio representativo de la transición de la construcción entramada y la introducción de los nuevos materiales: las columnas de fundición y las vigas metálicas. Su estudio permite recrear el proceso constructivo original y situar los daños más frecuentes en la mayor parte de los edificios anteriores a 1900.

Palabras clave: Entramado de madera; estructuras metálicas; proceso constructivo; intervención; Madrid; contrato de obra.

\section{ABSTRACT}

The study of buildings that have been preserved carried out by an analytical approach to original construction contracts, together with several damaged structures checked, has led to an overall timber frame house's analysis. This approach could be divided into three main sections: first, traditional construction methods in Madrid from 1669 to nineteen hundred, within context. Next, a graphic analysis attending to information covered in construction contracts and the fact of being able to get information from buildings. Finally, it is shown an extended approach to case studies in buildings that have been preserved. This investigation is supported by a deep study of a particular corner of Madrid considered as a transition case study from timber frame systems to the introduction of iron columns and steel structures. Original construction method revealed could lead to focus on main pathologies of most of the houses built in Madrid before nineteen hundred.

Keywords: Timber frame buildings; steel beams; iron cast columns; traditional construction; heritage; Madrid archives.

Universidad de Alcalá. Alcalá de Henares (España)

Persona de contacto/Corresponding Author: esperanza.gonzalez@uah.es (E. González-Redondo)

Cómo citar este artículo/Citation: González-Redondo, E. (2014). De la construcción entramada a la introducción de estructuras metálicas en Madrid. Informes de la Construcción, 66(534): e025, doi: http://dx.doi.org/10.3989/ic.12.100.

Licencia/License: Salvo indicación contraria, todos los contenidos de la edición electrónica de Informes de la Construcción se distribuyen bajo una licencia de uso y distribución Creative Commons Reconocimiento no Comercial 3.o. España (cc-by-nc). 


\section{INTRODUCCIÓN}

$\mathrm{Al}$ igual que otras ciudades europeas (1), en Madrid quedan numerosos edificios construidos con entramado de madera que se encuentran en mal estado de conservación y cuya rehabilitación se hace necesaria (2). Dada la escasez de información existente en este campo, el objetivo inicial consistió en conocer documentalmente como se construían estas casas para disponer de una información gráfica y escrita previa a la intervención (3). La investigación de archivo, tan lejana a las actuales búsquedas de información es, por su propia naturaleza, minuciosa, lenta y con pocos resultados inmediatos, de ahí la dificultad del análisis. Ese recorrido comienza en el siglo XVI y finaliza con las construcciones de principios del siglo XX (4). Refleja, desde distintas perspectivas, los cambios que se fueron produciendo y permite recrear, con muy pocas variaciones, el proceso constructivo de la mayor parte de los edificios construidos en Madrid, Figura 1. La obtención de información gráfica previa a la intervención es de difícil acceso, en parte, porque no siempre existía. Su complejidad conduce, en la mayoría de los casos, a la elaboración de un levantamiento de los planos con pocos datos de partida disponibles. Una comprensión global de esta tipología constructiva, junto con el conocimiento de las dimensiones de los elementos estructurales y de sus sistemas de unión, previos a su representación gráfica, permite realizar un riguroso análisis de los daños observados, medidos y representados. Con ello se inicia la elaboración del diagnóstico final y facilita en gran medida los trabajos de intervención posteriores.

El Plan General de Ordenación Urbana de Madrid, PGOUM, 1997, a través del Catálogo de Edificios protegidos (5), establece distintas condiciones de catalogación, con distintos niveles y grados de protección para cada edificio antiguo. En los últimos años se ha hecho un recorrido de los barrios del Centro Histórico de Madrid, dentro de la Cerca de Felipe IV, realizando una toma de datos en numerosos edificios con entramado de madera que aún se conservan. Este análisis ha permitido cotejar la información contenida en los contratos de obra originales estudiados (Figura 2), con la realidad construida que se ha conservado. Junto a ello, se han estudiado los problemas más frecuentes en este tipo de construcciones determinando sus causas, que por falta de espacio serán objeto de estudio en otro trabajo, así como intervenciones previas en esos mismos edificios, algunas no muy afortunadas.

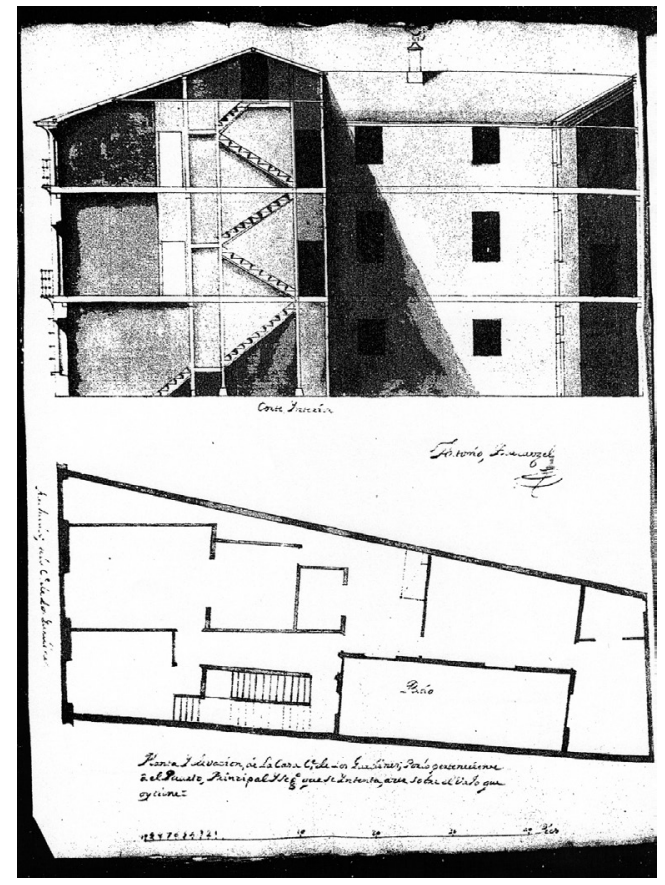

Figura 2. Contrato de obra: C/ de los Jardines, 1759 (AHPM 17.978).

A la vista de los estudios realizados, se puede avanzar que la base constructiva de la mayor parte de los edificios que se conservan, construidos antes de 1900 , ha sufrido muy pocos cambios.

\section{LA CONSTRUCCIÓN CON ENTRAMADO DE MADERA EN EUROPA}

La gran disponibilidad de los materiales para su construcción, un armazón de madera y un relleno de fábrica (ladrillo, adobe, mampostería y yeso, entre otros), su ventaja económica, así como la facilidad de su puesta en obra: ligereza y rapidez, han hecho que, desde antiguo, la construcción con entramado de madera se extendiera por todo el mundo, Figura 3. Las distintas variaciones de la configuración de los entramados, las escuadrías de las piezas de madera así como el material de relleno, han conducido a denominaciones diversas (6): Fachwerk en Alemania, sistema Pombalino en Portugal, himis en Turquía, colombage en Francia, Half-tim-
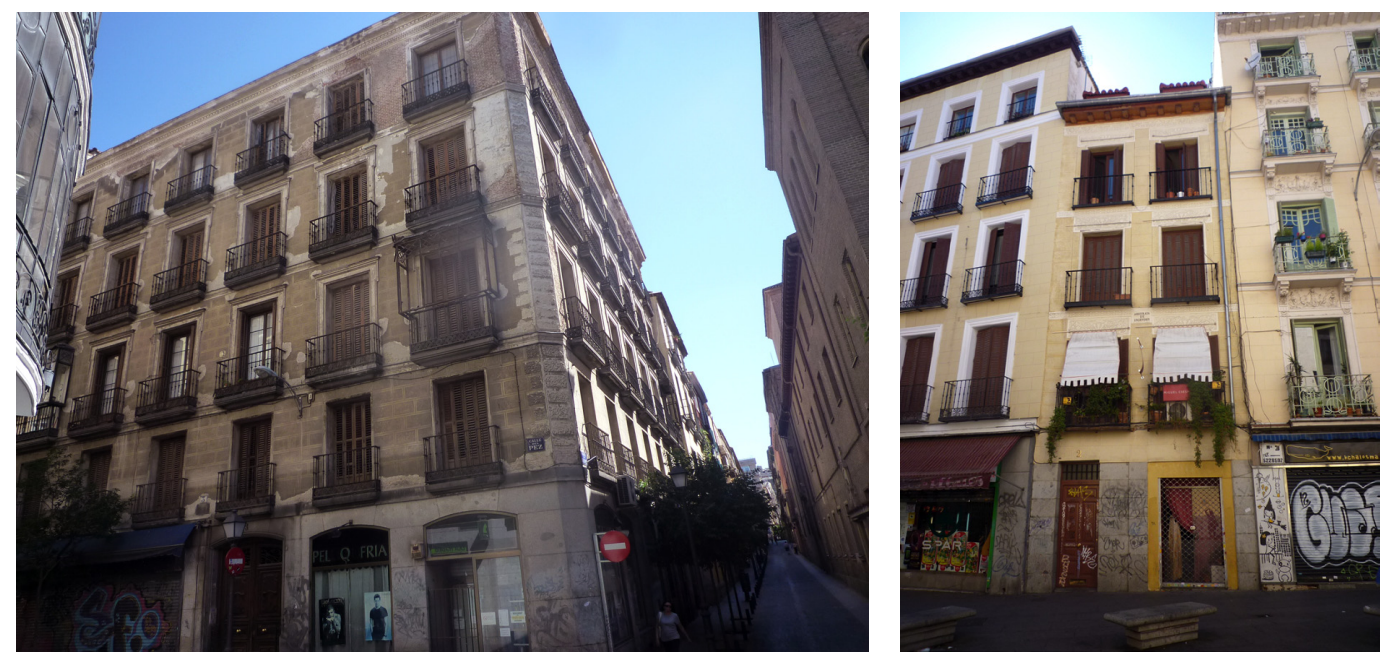

Figura 1. Casas construidas con entramado de madera en Madrid. 
bered en Inglaterra (7) o Casa baraccata en Italia (8); También se extiende por los países Escandinavos (9). En Madrid se denomina, desde antiguo, construcción con entramado de madera -Rejón de Silva, A. 1788 (10)-. En concreto, la gran altura de las edificaciones de Madrid, en comparación con las realizadas en otros países europeos, así como la extensión de su uso, desde los primeros documentos conocidos en el siglo XVI hasta principios del siglo XX, aportan características singulares.

Desastres naturales, como el caso del terremoto de Lisboa en 1755, que fue la causa de la introducción del sistema Pombalino (12), o la vulnerabilidad de estas construcciones frente a los incendios, como ocurrió tras el tercer incendio de la Plaza Mayor de Madrid en 1790 (13), han marcado, en ciertas regiones, un punto de inflexión en la evolución de los sistemas constructivos con entramado de madera. La extensión de esta forma de construir en áreas con elevado índice de riesgo sísmico y la constatación de que muchas han permanecido en pie mientras construcciones modernas de hormigón armado han colapsado, ha llevado a numerosos estudios recientes en estas áreas sísmicas -Turquía (14) y (15), Grecia (16) e Italia (8)- Los resultados han determinado las ventajas del comportamiento de la construcción entramada frente al colapso al adaptarse mejor a los movimientos sísmicos, aspecto éste que ya aparecía recogido en tratados como Clairac y Sáenz (1877) (17) y Ger y Lóbez (1898) (18), entre otros.

\section{LA CONSTRUCCIÓN EN EL CENTRO HISTÓRICO DE MADRID}

La construcción en el Centro histórico de Madrid, al igual que en otras ciudades históricas, se va adaptando a una trama urbana ya existente. En este caso, ya queda reflejada en el Plano de Texeira (1656) y va creciendo en altura y colmatando espacios libres dentro de la cerca de Felipe IV hasta principios de 1900. El comienzo de los contratos de obra referidos en este trabajo data de esa primera información topográfica. Se revisa, a través de casos concretos, la construcción de edificios de nueva planta en el mismo lugar de otros demolidos y dentro de manzanas con siglos de historia que se conservan en nuestros días, (Figura 2). Deja para estudios posteriores la construcción que tuvo lugar a partir del Plan Castro (1860) (19), con la extensión de los nuevos barrios, así como el análisis de edificios singulares con condiciones especiales de catalogación y ampliamente documentados (20). Atendiendo a la posibilidad de realizar un estudio constructivo en detalle, se puede establecer una línea divisoria de los proyectos y edifi- cios estudiados hasta la fecha, en tres períodos: a) 1669-1769, b) 1769-1847 y c) 1847-1900, (21).

La datación de estas casas y el análisis de las variaciones de este sistema constructivo, es un trabajo en curso. Hasta la fecha, un estudio global ha permitido determinar los cambios constructivos que se han ido produciendo y junto a ello situar los daños más frecuentes. En este punto, dadas las propias características de la metodología de investigación empleada, el análisis ha encontrado los siguientes obstáculos: es difícil encontrar edificios que simultáneamente: a) estén documentados gráficamente: plantas, alzados y en su caso secciones; b) que exista un contrato de obra que refleje las condiciones de ejecución de la obra y los materiales empleados; y c) que el edificio objeto de estudio se haya conservado en su totalidad, (nivel de protección estructural o integral). El caso que aquí se analiza con detenimiento, participa de los tres aspectos y por ello se utilizará como base para explicar la tradición constructiva previa y los nuevos cambios introducidos. Junto a ese caso particular se revisan otros edificios, algunos han desaparecido, bien por la apertura de la Gran Vía u otras vías; en otros casos se ha levantado un edificio nuevo, o se han añadido nuevas alturas a edificios existentes y otros, con protección parcial, han sido demolidos y tan sólo se ha conservado la fachada.

\section{LAS ESTRUCTURAS DE ENTRAMADO DE MADERA EN MADRID}

La mayor parte de los edificios de Madrid se levantaban sin un contrato de obra, o no es fácil llegar a encontrarlo. Para la obtención de la licencia tan sólo se obligaba a presentar un esquema del alzado y generalmente, se adjuntaba también un plano de la planta baja o de la principal. Esta tradición, que se puede constatar en el Archivo Histórico de Madrid (AVS), se acompañaba de una sencilla y breve memoria constructiva que con frecuencia se repetía para todos los edificios. Por ello, la consulta de estos documentos, si bien relevante, no permite progresar en la investigación.

El análisis de los contratos de obra encontrados en el Archivo Histórico de Protocolos de Madrid (AHPM), ha permitido avanzar en una investigación que apenas se refleja en la bibliografía moderna existente y que se presenta como herramienta esencial para la intervención. Se realizó con estructura portante de madera tanto horizontal como vertical, denominado entramado -Clairac y Sáez, 1877 (17) y Bails, 1802 (22)-, sobre una cimentación y planta baja construida
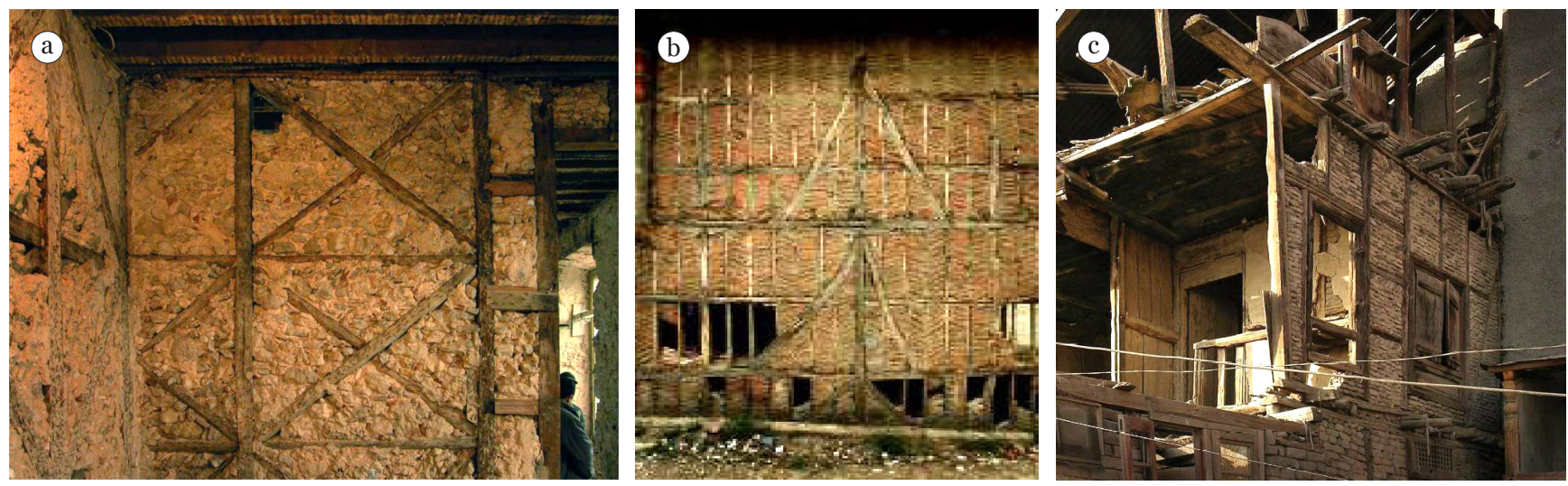

Figura 3. La construcción entramada en otros países: a) Sistema Pombalino (Portugal) (11), b) Himis en Turquía (8), c) dhajji Dewari en Srinagar (8). 
en su totalidad en albañilería (ladrillo o mampostería) que en el caso de existir tiendas solía resolverse con planta baja de cantería para el muro de fachada. Los muros de traviesa y medianeros se construían entramados con pies derechos sobre dados de granito. Sobre esta planta baja se levanta una estructura de madera desde los denominados «cuartos principales» hasta la realización de la cubierta con armaduras y entablados (Figura 4). El criterio rector en el dimensionado de estos entramados viene determinado por el ancho de sus carreras y sobre la base de ellas se dimensionaba el resto del armazón de madera que disminuye de sección a medida que el edificio crece en altura (4). Se construían entramados los muros de fachada a la calle, muros de fachadas interiores o patios, cítaras o muros de traviesa, medianerías y paredes delgadas interiores o tabiques. Los huecos o paños verticales resultantes de la separación de los maderos entre sí, se rellenaban de diversos materiales: ladrillo, mampostería, cascote, o adobe, (Figura 5). Análogamente, los entramados horizontales o forjados, también resueltos con estos mismos marcos o escuadrías de madera, se resolvían con maderos de secciones decrecientes según las luces a cubrir. Estos mismos marcos de madera, con su denominación y escuadría: canto, tabla y largo perviven durante tres siglos (4). La consulta de las publicaciones más relevantes permite conocer las características generales de esta forma de construir desde mediados del s.XIX, entre otros, García Gómez, 1854 (23), Marcos y Bausá, 1879 (24) y Ger y Lóbez, 1898 (18).

El proceso de sustitución gradual de esta construcción entramada por fábrica de albañilería tiene sus comienzos a finales del s. XVIII y el detonante hay que buscarlo en el incendio de la Plaza Mayor de Madrid en 1790. En esta ocasión se incendiaron 54 casas y no fue posible frenar su propagación hasta que el avance del fuego a lo largo de la hilera de casas llegó hasta una cuyas medianerías estaban construidas en su totalidad de fábrica. Este hecho lleva a los técnicos a reflexionar sobre la precariedad de los sistemas constructivos que venían utilizándose hasta el momento, en materia de protección con- tra incendios, y a replantearse posibles mejoras (13). Durante el s. XVIII son numerosas las referencias al respecto pero, en ningún documento encontrado hasta la fecha, queda reflejado el carácter de obligatoriedad de construir con albañilería. En la segunda mitad del s. XIX, comienza a recogerse todo un conjunto de normas más concretas (25). Con carácter general, a pesar del tiempo trascurrido desde los primeros sistemas constructivos de entramado de madera documentados en el s. XVII, hasta que concluye el proceso de sustitución por nuevas técnicas a principios del $\mathrm{s}$. XX, las soluciones constructivas llevadas a cabo durante estos tres siglos responden a un esquema análogo, con unas técnicas heredadas muy similares y que todavía en nuestros días, a pesar de las numerosas intervenciones, mantienen los marcos de madera originales, su disposición y la lógica del sistema constructivo original (4) y (21).

\section{DE LA CONSTRUCCIÓN ENTRAMADA A LA INTRODUCCIÓN DE LAS ESTRUCTURAS METÁLICAS: ANÁLISIS CONSTRUCTIVO}

El estudio de las construcciones con entramado de madera es complejo, ya que las estructuras de madera que se han conservado, muros, forjados y cubiertas, forman parte de estructuras interiores: It's hard to imagine how in Madrid, most of historical buildings, which from the front look like standard masonry buildings are, in fact, in the inside almost completely timber frame structures (Langenbach, C. 2007) (6). Se puede observar esta tipología constructiva tras la demolición de los edificios colindantes, al quedar sus medianerías al aire (Figura 6), o en edificios en fase de intervención, al picar los yesos que recubren los entramados.

\subsection{Metodología de trabajo: análisis del proceso constructivo}

Se desarrolla el estudio del caso particular de una esquina, que en su origen tenía una única casa (Figura 7), y que se
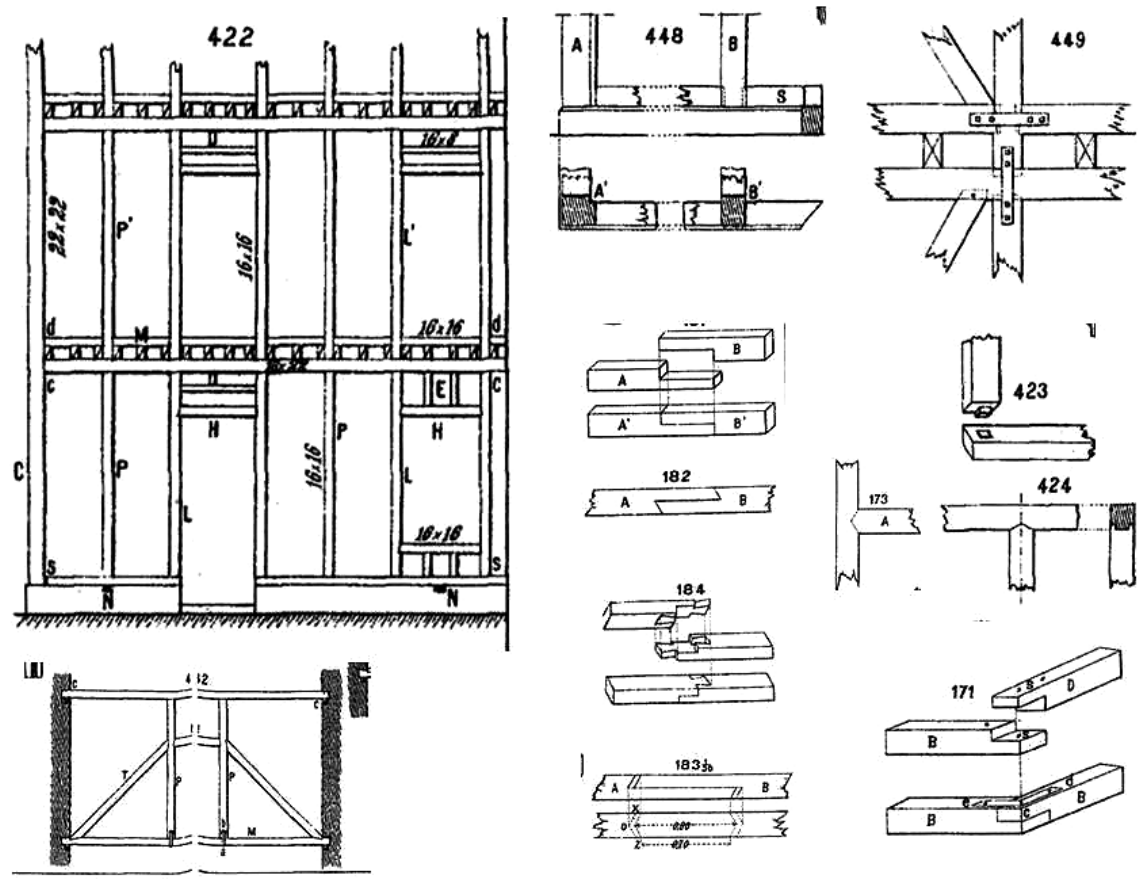

Figura 4. Construcción de paredes entramadas:

elementos principales y sistemas de unión, (Ger y Lóbez, 1898). 

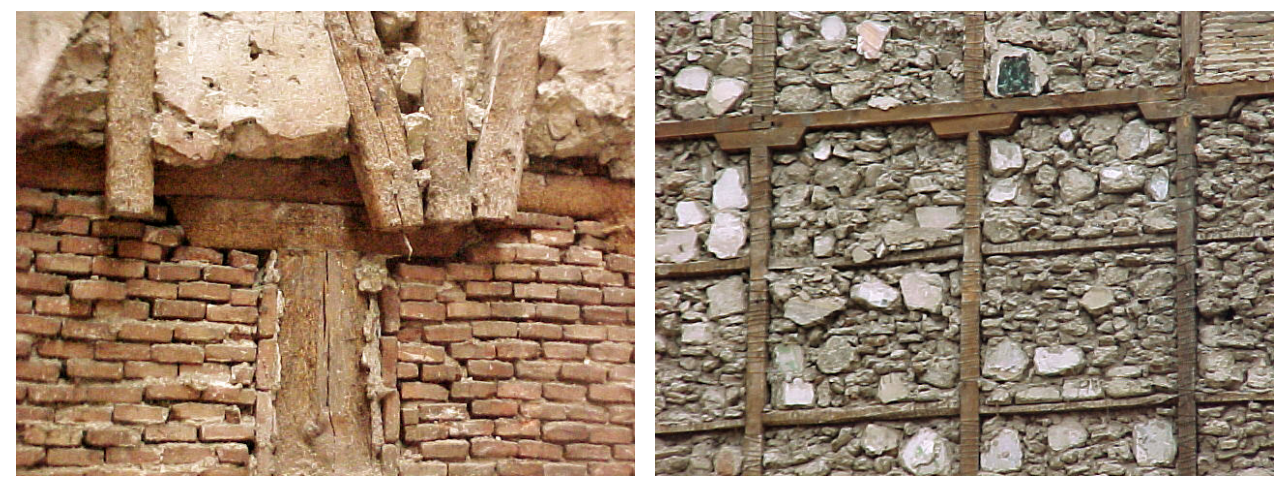

Figura 5. Detalles constructivos de entramado de madera en Madrid.
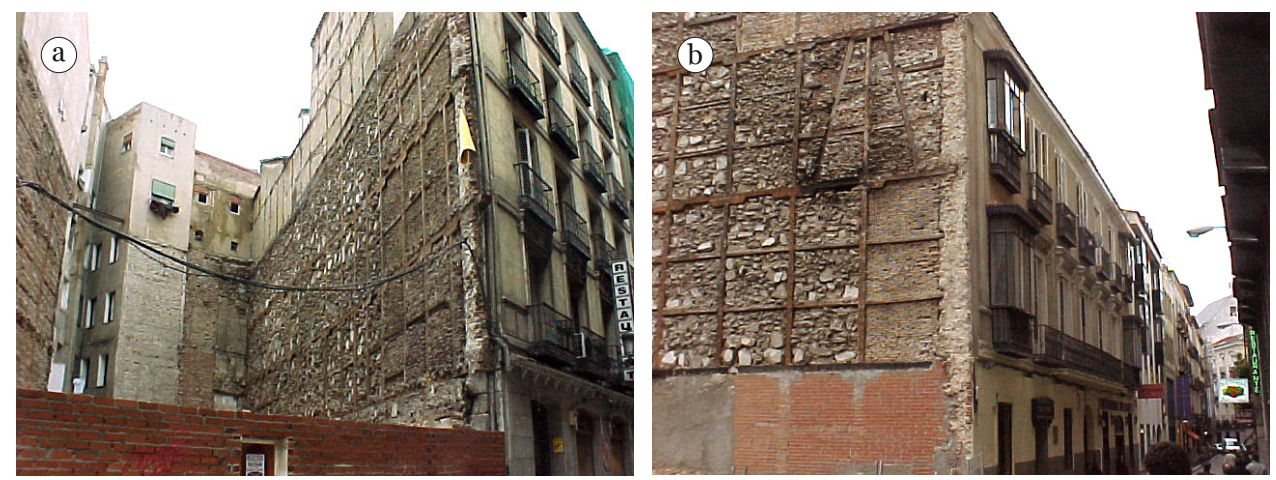

Figura 6. Paredes medianeras construidas con entramado de madera:

a) c/ Ventura de la Vega, c) c/de la Cruz.

compone actualmente de tres edificios independientes construidos en 1899. Es un proyecto de transición extensamente documentado ( $A H P M n^{\circ} 38.028$ ) (26), que cierra una etapa donde la tradición constructiva era la construcción entramada y que marca el inicio de los nuevos cambios. Se conservan las estructuras de madera para los muros de los patios, los muros de traviesa así como para algunos forjados y cubiertas. Al mismo tiempo, se introducen nuevos materiales: las columnas de fundición, las viguetas metálicas y las escaleras de hierro. Junto a ello, se incluyen notables mejoras que no se consideraban en fecha anterior, entre otras, la entrada de agua en cocinas y aseos, luz eléctrica, la consideración de construir un ascensor (aunque no se realizó) y la inclusión de portería y casa del portero. El análisis constructivo del proyecto seleccionado se presenta como modelo de referencia para estudiar otros con características muy similares y permitirá trasladar el análisis a la comprensión de la mayor parte de los edificios que todavía quedan en el Centro Histórico de Madrid. Estos datos facilitan el levantamiento de los planos y la elaboración de un diagnóstico previo a una futura intervención.

\subsection{El proyecto: documentación gráfica y escrita}

El proyecto anterior tenía una única casa que, según consta en el contrato de obra, pertenecía a la antigua manzana número 482, con números 1, 2, 14 y 15. Esta información se puede contrastar con la información histórica del plano de Espinosa de los Monteros (1759) y con el Plano de Ibáñez Ibero (1872), Figura 7. También se incluye que, antes de la demolición del edificio existente, estuvo prevista su rehabilitación. El conjunto del proyecto de nueva construcción del Arquitecto Benito González del Valle, en 1899, comprende tres casas: una grande en esquina, Pez 36, y otras dos pequeñas, Pozas
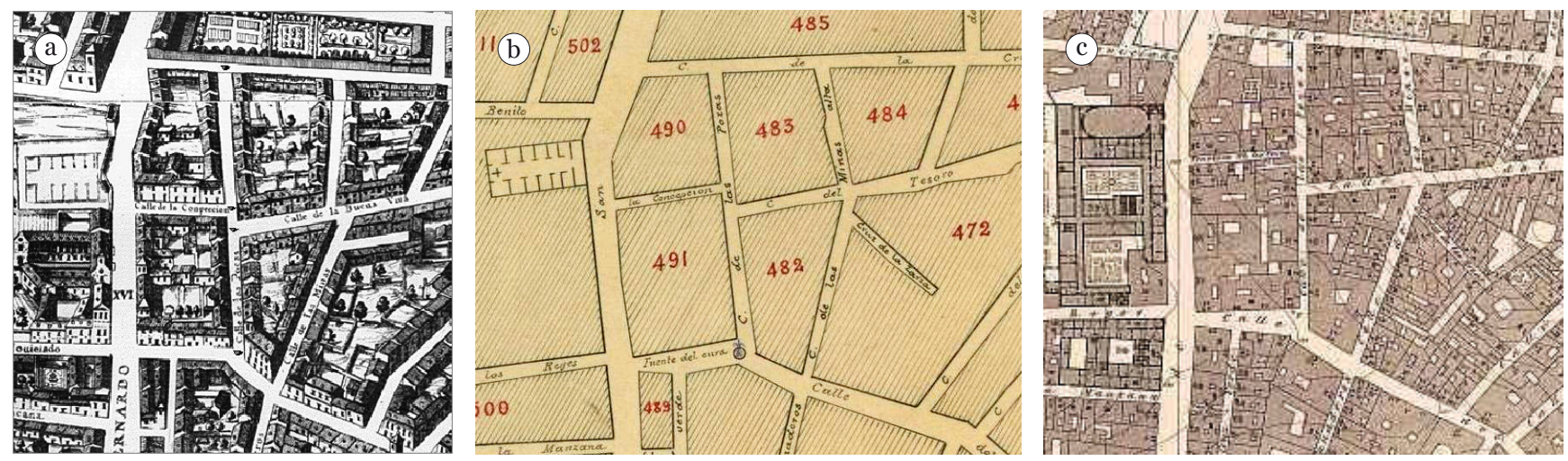

Figura 7. Planimetría histórica de la C/ del Pez c/v Minas c/v Pozas: a) Plano de Texeira (1656),

b) Plano de Espinosa de los Monteros (1759) y c) Plano Topográfico de Ibáñez Ibero (1872). 
4 y Minas 3. La documentación existente comporta un texto manuscrito de 54 páginas y una información gráfica de 5 planos: alzados, planta de sótanos, planta baja y planta principal (26). Según los planos de que disponemos y las vistas aéreas actuales, el edificio se corresponde con las trazas originales.

La casa grande, Pez 36, es un edificio complejo en esquina. Se organiza con cuatro crujías paralelas a fachada dejando en la segunda numerosos cuartos sin ventilación directa, una escalera principal singular de cinco tramos con descansillo compartido en el tercero y salida a dos tipos de viviendas, exteriores e interiores. Tiene condición de catalogación Integral (número 468). Consta de planta de sótanos vaciada en toda la superficie; planta baja distribuida en diez tiendas con sus correspondientes trastiendas, aseo y habitación vividera en tres de ellas, habitación del portero, portal, patios y escalera; plantas de entresuelo, principal, segundo y tercero, divididas en seis viviendas cada una (cinco exteriores y un interior) y planta de cubiertas con azoteas y buhardillas. Las casas pequeñas, Pozas 4, con condición de catalogación Integral (número 469) y Minas 3, con condición de catalogación Estructural (número 467), se componen de planta de sótanos en primera crujía, planta baja, plantas principal, segunda y tercera, divididas cada una en dos viviendas, y planta de cubiertas. Tienen cinco líneas de huecos en fachada y cuatro crujías paralelas a fachada, Figura 8.

\subsection{Trabajos previos: Vaciado de sótanos, replanteo y apertura de zanjas}

Según era costumbre, sólo se construían los sótanos en la primera crujía (21). Tenían iluminación y ventilación en la fachada y la escalera de bajada arrancaba desde la segunda crujía, como en la casa pequeña Pozas 4. Por primera vez, un contrato se refiere al vaciado de toda la planta de sótano en la casa grande, Pez 36. En la línea de medianería con las casas existentes se deja una banqueta de terreno ataludado de $1,50 \mathrm{~m}$ de ancho en su parte superior, para evitar perjuicios a las fincas colindantes, no se realiza el desmonte hasta que se acometen las traviesas y se hacen los entrepaños por puntos. El suelo de los sótanos se fija a una profundidad de 3,50 m por debajo de la rasante de la calle en la esquina de la c/Pez c/v Pozas. Realizados el vaciado y la nivelación, se ejecuta el replanteo general de los muros de fachada y de los muros interiores colocando al mismo tiempo puntos invariables para determinar los anchos de las zanjas que llevan $7 \mathrm{~cm}$ de zarpa a cada lado del grueso de los muros de sótanos.

\subsection{Cimentación}

Fray Lorenzo de San Nicolás (1639) (27) establece los primeros criterios de dimensionado de la cimentación, el ancho que debe tener la zanja y su profundidad, indicando que, «al cimiento se le ha de dar la octava parte de su grueso a cada lado, se deberán realizar los trabajos de vaciado hasta alcanzar el firme añadiendo por cada lado de la zanja corrida medio pie, unos $14 \mathrm{~cm}$, con respecto al ancho o espesor del muro que ha de levantarse». Relativo al fondo, Juan de Torija (1661) (28) indica que, «han de tener la cuarta parte de la luz de la crujía a cubrir y siempre y cuando se haya alcanzado el firme», de este modo, un cimiento de 3 pies, cubrirá una crujía de unos 12 pies. En las Ordenanzas de 1892 (25), en su artículo 723, establece que, «cuando el terreno firme se encuentre próximo a la rasante de la calle, la fundación del cimiento de los muros que linden con la vía pública no podrá tener menos de un metro de profundidad».
En este proyecto, todos los cimientos se realizan a una profundidad de 1,50 m por debajo del nivel que se ha indicado para pavimento de sótanos. Si el terreno fuera de mala calidad, se macizarían las zanjas del cimiento con fábrica de ladrillo recocho, vitrificado, sentado por hiladas a nivel con mortero común o hidráulico en toda su altura. El resto de las zanjas se macizan con pedernal vivo, ladrillo, ripio y mortero, perfectamente apisonado, no debiendo exceder las tongadas de pedernal $35 \mathrm{~cm}$ de altura. En los pasos de las atarjeas por los muros del cimiento se hacen las pilastras y roscas de ladrillo. La investigación realizada desvela que el proceso de relleno de las zanjas de cimentación no ha variado desde las indicaciones de S. Nicolás en 1639 (27), hasta el contrato aquí mencionado, 1899.

\subsection{Construcción de sótanos: introducción de estructuras metálicas}

Los muros de los sótanos se construyen con fábrica de ladrillo recocho y mortero de cal y arena excepto los que están enterrados por una de las caras, que se construyen y se revisten con cemento (aparece el cemento mencionado por primera vez), van guarnecidos y blanqueados con yeso o con cal y se dejan los huecos para las bajadas. En el portal y en el suelo de las tiendas se abren lumbreras de derrame con rejillas que permiten la ventilación cruzada en fachadas y patios hasta nivel de sótano. Los muros de la primera traviesa se componen de gruesas pilastras a plomo de las columnas de planta baja unidas con arcos de medio punto o rebajados. El resto de los huecos se cubren con arcos adintelados o de medio punto, utilizando mortero hidráulico, arena de río y cemento lento.

El techo de los sótanos se forma con vigas de hierro de doble $T$ de $18 \mathrm{~cm}$ de altura, distanciadas entre ejes 50-60 $\mathrm{cm}$ y van apoyadas sobre unos llantones de hierro $(10 \mathrm{~cm} \times 1 \mathrm{~cm})$ colocados sobre los muros. El forjado se realiza con bovedillas dobles de rasilla hueca y mortero hidráulico. Se ponen brochales y escuadras de hierro sujetas con tornillos y umbrales en los huecos. Debajo de los tabicones de los portales y en los puntos de arranque de las escaleras se doblan las vigas y se unen con pasadores. Tradicionalmente estos sótanos o cuevas se cubrían con bóvedas de cañón de ladrillo colocados de canto o sardinel sobre camón.

\subsection{Planta baja de cantería: las tiendas y los patios}

Desde los primeros contratos de obra estudiados en 1669 (21), se indica que, rellenas las zanjas de cimentación, se colocaban los sillares de piedra Berroqueña de media vara de lecho, $42 \mathrm{~cm}$, y media vara de alto. Sobre ellos se levantaban los muros de mampostería enrasándolos todos a nivel siendo, en fecha posterior, enteramente de ladrillo. Cuando se construían tiendas en planta baja se levantaba todo el muro de cantería, como en este caso que la fachada a la Calle del Pez tiene sillares con pilastras de piedra granítica en toda la altura de planta baja. Las fachadas de la casa grande por las calles de Minas y Pozas y los de las casas pequeñas, se construyen con sillares lisos de la misma piedra, y también son lisos los sillares de ángulo que tienen un chaflán de $42 \mathrm{~cm}$. La hilada inferior de dicha cantería es de losas de elección también de granito de $35 \mathrm{~cm}$ de grueso con sus zarpas correspondientes. La parte superior de dichas losas queda enterrada $20 \mathrm{~cm}$ por debajo de la rasante de las calles respectivas. Las pilastras tienen las cajas necesarias para el apoyo de las vigas y carreras 
y los sillares de ángulo y los de medianería de las tres casas también, para las bajadas de las aguas pluviales.

Los patios de la casa grande tienen un zócalo de granito de $70 \mathrm{~cm}$ altura que cuaja todo el espesor del muro y los de las casas pequeñas tienen antepechos de la misma altura. Todos los huecos de fachadas y de salida a los patios, tienen unos batientes de piedra berroqueña y también es de granito el primer peldaño de todas las escaleras. Los patios de la casa grande tienen un solado de piedra berroqueña colocado a junta perdida. Las basas para los pies derechos son también de granito de $70 \mathrm{~cm}$ de altura y su grueso varía según el entramado. Todas las columnas de planta baja descansan sobre losas de $(70 \times 56 \times 30 \mathrm{~cm})$ y la cara superior de estas basas queda enrasada a nivel con el piso, Figura 8.

\subsection{Fábrica de ladrillo en fachadas y cerramientos}

El muro de fachada de la casa grande, Pez 36, se construye con fábrica de ladrillo de $98 \mathrm{~cm}$ de espesor en la planta de entresuelo y se va retallando en los superiores hasta terminar con $70 \mathrm{~cm}$ en el tercero; y el de las pequeñas tiene $84 \mathrm{~cm}$ y disminuye hasta $56 \mathrm{~cm}$. Tiene un frente exterior de ladrillo fino de la Ribera, con piezas aplantilladas y de tres cuartos de pie (Figura 9). La traba es a la española y no se admite el enripiado interior sino sólo ladrillo entero y medio. El contrato indica que se tendrá particular esmero en la colocación de los ladrillos que forman el dibujo que decora la fachada. En la actualidad todos los elementos de la fachada están protegidos, (Catálogo de elementos protegidos, PGOUM, 1997) (5). Los salmeres que sirven de arranque a los arcos de los huecos de entresuelo, así como la arquería que remata la fachada por debajo del alero, están revestidos de cemento y pintados fingiendo piedra blanca. Al llegar la fábrica a la altura de los pisos respectivos, se colocan los nudillos $(56 \times 20 \times 14 \mathrm{~cm})$ a una distancia de 1,40 m entre ejes, y se reciben con yeso, lo mismo que las carreras.

Tradicionalmente los muros medianeros se construían con entramado de madera (Figura 5 y Figura 6), a pesar de la recomendación de abandonar esta solución para evitar la propagación de incendios (13). Esta técnica va desapareciendo, y este caso marca esa tendencia, los muros de medianería de las tres casas se construyen con fábrica de ladrillo recocho, con cadenas de cemento de cuatro hiladas en cada metro, y tienen un espesor de $42 \mathrm{~cm}$ en toda su altura. Sin embargo, incluso a pesar de su prohibición en las Ordenanzas de 1892, contratos de obra posteriores recogen que siguieron construyéndose, y es frecuente verlos en Madrid.

\subsection{Las columnas de fundición y las vigas de fachada}

El caso aquí estudiado, también en este punto, marca el inicio de un cambio que se va generalizando en construcciones posteriores. En planta baja y como muestran los planos, se colocan columnas de fundición de $18 \mathrm{~cm}$ de diámetro exterior por debajo del collarino y de $3 \mathrm{~cm}$ de grueso. En las embocaduras de las alcobas se colocan también columnas de fundición de $10 \mathrm{~cm}$ de diámetro y de $25 \mathrm{~mm}$ de grueso. Todas ellas llevan sus correspondientes basas, capiteles, botoneras y zapatones con ménsulas sujetas a las carreras con placas y tornillos. La carencia de detalles constructivos en los contratos de obra originales puede suplirse con la consulta de las figuras que recogen los tratados de construcción más relevantes de la época, entre otros, Ger y Lóbez 1898 (18), Figura 10.

En su origen, los umbrales de fachada y los huecos de paso en los muros se resolvían con elementos de madera. Posteriormente, y así se constata tras el reconocimiento de muchos edificios, la solución más extendida era una cara exterior resuelta con arcos adintelados de ladrillo y una interior con umbrales de madera que servían también de apoyo a los forjados de madera. Esta casa marca también esa transición, sobre los huecos de fachadas en planta baja y trasdosando la sillería, se colocan vigas armadas en forma de umbrales. Se componen de dos vigas de doble $T$, de $20 \mathrm{~cm}$ de altura, con un añadido de tercia aplantillada sujeta a las barras con pasadores de tuerca. La barra de intradós enrasada a los haces del muro recerca sin solución de continuidad todo el aro de fachada. Se une a las carreras de medianería con escuadras y tornillos y se refuerza en los ángulos de fachada con escuadras especiales. Paralelamente a este umbral y próxima al paramento de fachada se coloca una viga de doble $T$ de $16 \mathrm{~cm}$ que contiene las repisas fingidas de los entresuelos y se apoya sobre palomillas de hierro, Figura 10.
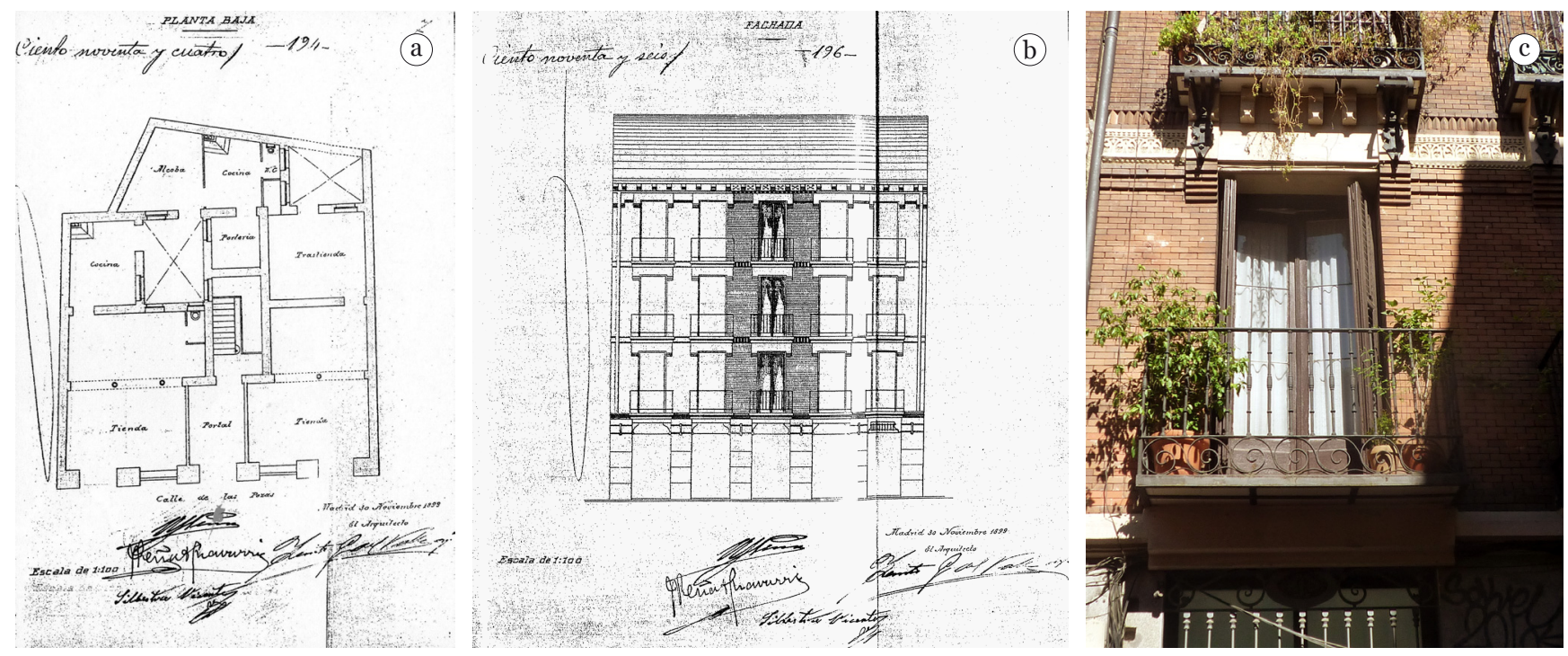

Figura 8. Casa en Pozas 4: a) Planta baja, b) Alzado (AHPM no 38.028) y c) Fachada. 

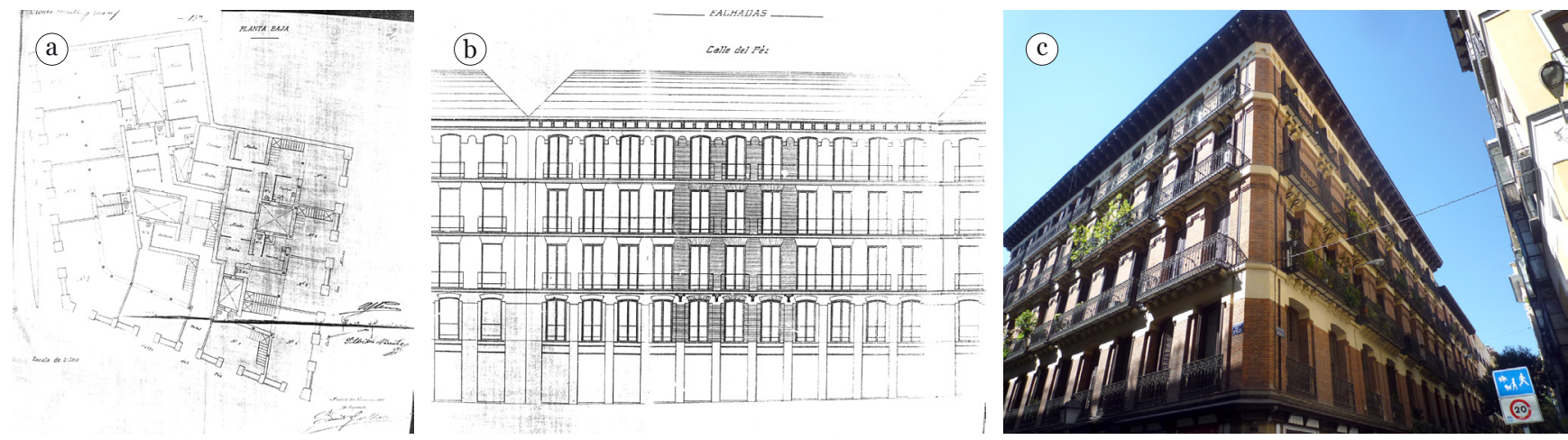

Figura 9. Casa en Pez 36: a) Planta baja, b) Alzado (AHPM no 38.028) y c) Fachada.
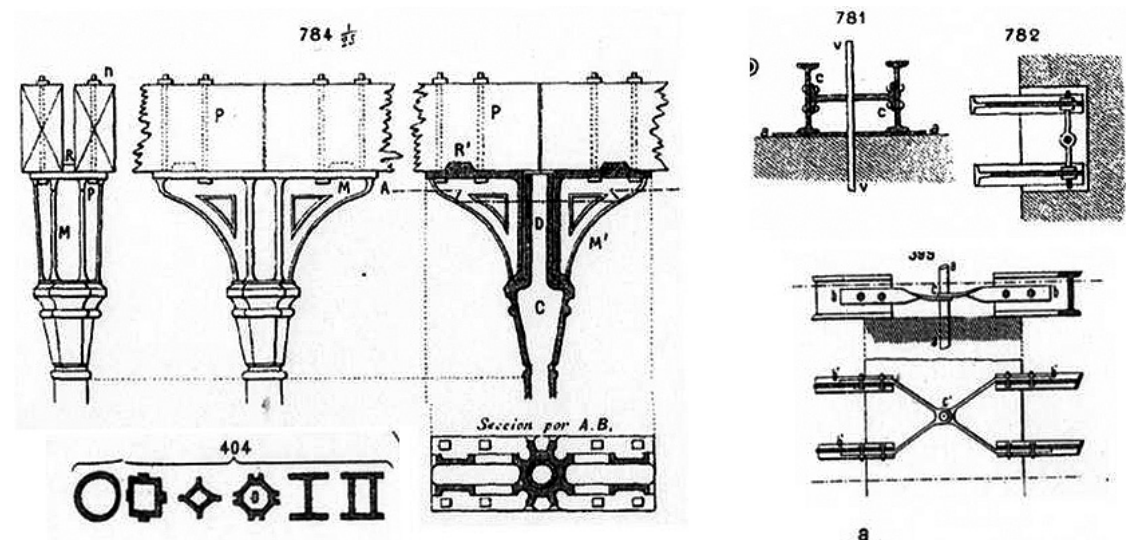

EV
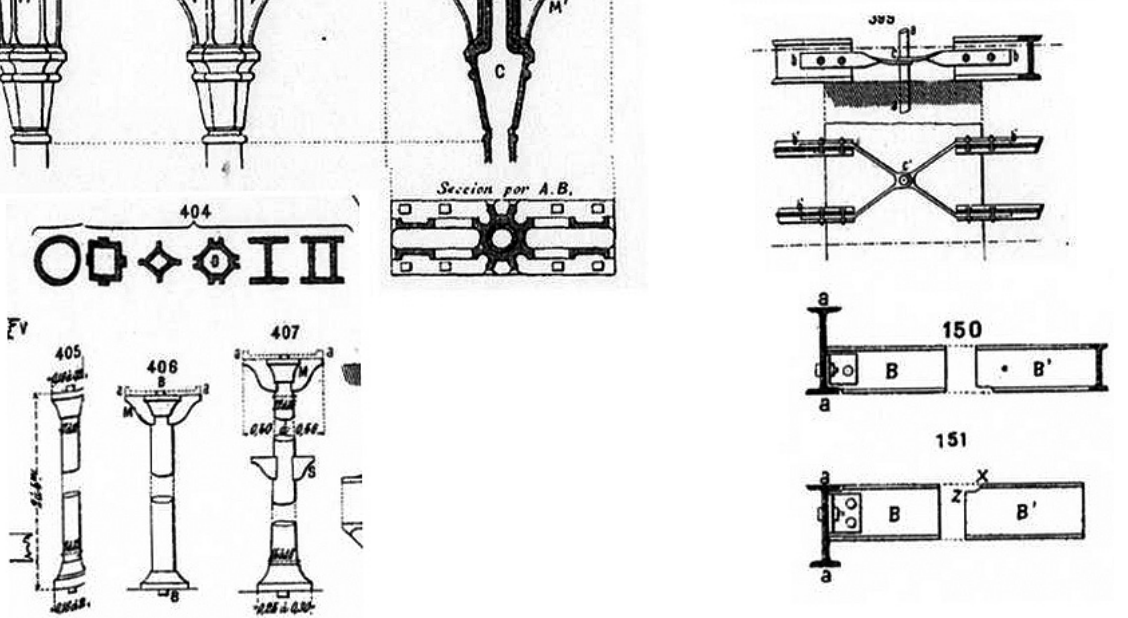

151

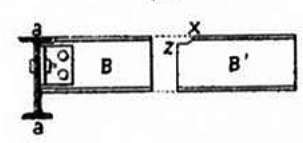

Figura 10. Estructuras metálicas: columnas de fundición y vigas de fachada (Ger y Lóbez 1898).

Los umbrales de los sótanos son también de vigas de doble $T$ de $18 \mathrm{~cm}$ en las cuales van roblonadas las vigas de piso formando los tragaluces por debajo de los escaparates de las tiendas en donde se colocan vigas de doble $T$ de $8 \mathrm{~cm}$. En la cantería se hacen las cajas necesarias para embeber las cabezas de las vigas y los techos emplomados. En las casas pequeñas, los umbrales de planta baja se forman con dos vigas de doble $T$ forjando el entrevigado. La viga del intradós corre en toda la línea de fachada y se empalma con las carreras de medianería. En la Tabla 1 se muestran las estructuras metálicas introducidas en este proyecto, su posición y sus dimensiones.

\subsection{Entramados verticales de madera}

Aunque las secciones de las piezas de madera utilizadas en los entramados quedan recogidas en los contratos de obra, al menos desde 1669, por primera vez, dentro de la investigación realizada, se encuentra un plano en el que aparecen reflejadas gráficamente las dimensiones y la colocación de los pies de derechos de madera en la planta (Figura 11). Los entramados verticales arrancan del piso entresuelo y son de tercia en éste y en el principal y de sesma en el segundo y tercero, en las tres casas. Estos entramados llevan dos órdenes de puentes de $10 \mathrm{~cm}$ que cuajan todo el grueso del entramado, van recibidos a espera y sujetos con clavos. Una vez entomizada la madera se tabica todo el grueso de los cajones con ladrillo recocho y yeso negro.

Las carreras son enterizas del marco correspondiente a cada entramado y se indica que su largo no debería ser menor de 7,0 m. Las uniones van contrapeadas y con ensambles a lazo, media madera o cola de milano (Figura 4). Todos los empalmes llevan gatillos y escuadras. En las embocaduras de las alcobas se doblan las carreras. Las carreras exentas de la primera traviesa, que van sobre columnas y atraviesan el portal, van armadas de dobles vigas reforzadas de $30 \mathrm{~cm}$ de altura (Figura 10); se añade un pie y cuarto aplantillado en la casa grande y $20 \mathrm{~cm}$ de tercia en las pequeñas. Los tabiques sencillos también se construyen entramados (Figura 4), van armados al aire con un madero horizontal apoyado sólo en dos puntos que lo sostienen, las aldavias, van entomizados y se rellenan de ladrillo recocho y yeso. Los elementos de unión que se utilizan son clavos, gatillos y barras de hierro. Los clavos que sujetan los entramados se emplean en todos los encuentros de carreras, empalmes de los entramados horizontales, verticales y oblicuos, y campanas de chimeneas. También se colocan gatillos y barras de hierro atendiendo a la forma y dimensiones según su colocación (Figura 4). 
Tabla 1. Estructuras metálicas: vigas metálicas y columnas de fundición.

\begin{tabular}{|l|l|l|c|}
\hline \multicolumn{3}{|c|}{ Estructuras metálicas } \\
\hline \multirow{4}{*}{ Posición } & Elementos constructivos & Perfil & Luz/altura \\
\hline \multirow{4}{*}{ Fuecos de fachada } & Hueco de planta baja (umbrales en tiendas) & 2 doble T $(20 \mathrm{~cm})$ & $2,2 \mathrm{~m}$ \\
\cline { 2 - 4 } & Entresuelo (umbrales) & 1 doble T $(16 \mathrm{~cm})$ & $1,4 \mathrm{~m}$ \\
\cline { 2 - 4 } & Sótano (umbrales) & 1 doble T $(18 \mathrm{~cm})$ & $1,4 \mathrm{~m}$ \\
\cline { 2 - 4 } & Tragaluces (sótano-tiendas) & 1 doble T $(8 \mathrm{~cm})$ & $0,8 \mathrm{~m}$ \\
\hline \multirow{2}{*}{ Tiendas } & Forjado de sótano & doble T $(16 \mathrm{~cm})$ & $5,0 \mathrm{~m}$ \\
\cline { 2 - 4 } & Forjado de entresuelo & doble T $(16 \mathrm{~cm})$ & $5,0 \mathrm{~m}$ \\
\cline { 2 - 4 } & Forjados de baños y cocinas & doble T $(16 \mathrm{~cm})$ & $5,0 \mathrm{~m}$ \\
\hline
\end{tabular}
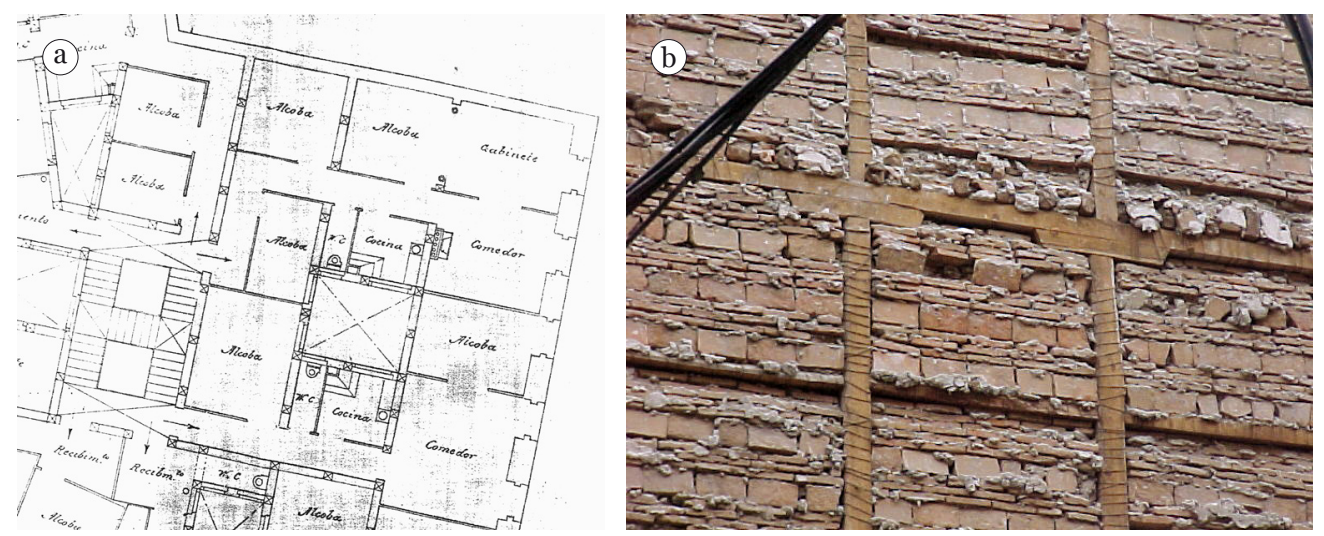

Figura 11. Muros entramados: a) Detalle de la planta tipo de Pez 36 y b) Detalle de entramado de madera.

Estas estructuras de madera, que aparentemente se levantaban sin un cálculo previo, han demostrado tener unas dimensiones acertadas. Prueba de ello es que numerosas casas con una antigüedad de más de 180 años los han conservado. En la Tabla 2, se muestra la solución constructiva de los distintos muros, desde los sótanos hasta la planta $5^{\mathrm{o}}$. Junto a ello, se indican sus dimensiones principales y los materiales utilizados: sillería, fábrica de ladrillo, columnas de fundición y entramados de madera.

\subsection{Estructura horizontal: hierro y madera}

Los pisos de los sótanos de las tres casas se construyen con vigas de hierro de doble $T$ de $16 \mathrm{~cm}$ de altura, separadas de 50-70 cm entre ejes, según los casos (Figura 12). También son de hierro el piso entresuelo de la casa grande y los de todas las cocinas y retretes de las tres casas. El entrevigado se forja con dobles bovedillas de rasilla y cemento. En las azoteas tam- bién se colocan vigas de hierro con dobles bovedillas. Donde resulta necesario, se colocan brochales sujetos con escuadras y tornillos. Para las casas pequeñas el contrato indicaba que se volarían vigas de hierro para contener los balcones, en el caso de que no se resolviese hacerlos con armadura de repisa, como era la tradición, pero finalmente no se realizó. Los demás pisos, como venía siendo costumbre, se construyen con vigas de madera del marco apropiado a los vanos, sin embargo se abandona la solución tradicional de hueco por macizo (4) (Figura 12b) y en este caso se forjan con botes de barro cocido, siendo la primera vez que se menciona esta solución para el forjado. Tanto los pisos de hierro como los de madera van acodalados con dos o tres órdenes de zoquetes según la luz de las crujías.

\subsection{Las armaduras de madera}

La estructura de las cubiertas se resuelve con armaduras de par $y$ hilera y de par y picadero con sus pares y atirantados corres-

Tabla 2. Soluciones constructivas y dimensiones de los muros en Pez 36 (1899).

\begin{tabular}{|c|c|c|c|c|c|c|c|c|c|c|}
\hline \multicolumn{11}{|c|}{ Solución constructiva y dimensiones de los muros (1899) } \\
\hline Altura & \multicolumn{2}{|c|}{ Muro de fachada } & \multicolumn{2}{|c|}{ Muro de traviesa $1^{\circ}$} & \multicolumn{2}{|c|}{ Muro de traviesa 2, 3, 4} & \multicolumn{2}{|l|}{ Muros de patio } & \multicolumn{2}{|c|}{ Muros medianeros } \\
\hline Planta $3^{\mathrm{a}}$ & Fábrica de ladrillo & $70 \mathrm{~cm}$ & Entramado de sesma & $21 \mathrm{~cm}$ & Entramado de sesma & $21 \mathrm{~cm}$ & Entramado de sesma & $21 \mathrm{~cm}$ & Fábrica de ladrillo & $42 \mathrm{~cm}$ \\
\hline Planta $2^{\mathrm{a}}$ & Fábrica de ladrillo & $83 \mathrm{~cm}$ & Entramado de sesma & $21 \mathrm{~cm}$ & Entramado de sesma & $21 \mathrm{~cm}$ & Entramado de sesma & $21 \mathrm{~cm}$ & Fábrica de ladrillo & $42 \mathrm{~cm}$ \\
\hline Principal & Fábrica de ladrillo & $90 \mathrm{~cm}$ & Entramado de tercia & $28 \mathrm{~cm}$ & Entramado de tercia & $28 \mathrm{~cm}$ & Entramado de tercia & $28 \mathrm{~cm}$ & Fábrica de ladrillo & $42 \mathrm{~cm}$ \\
\hline Entresuelo & Fábrica de ladrillo & $98 \mathrm{~cm}$ & Entramado de tercia & $28 \mathrm{~cm}$ & Entramado de tercia & $28 \mathrm{~cm}$ & Entramado de tercia & $28 \mathrm{~cm}$ & Fábrica de ladrillo & $42 \mathrm{~cm}$ \\
\hline Planta baja & Cantería & $115 \mathrm{~cm}$ & Columnas fundición & $18 \mathrm{~cm}$ & granito $(70 \mathrm{~cm})+$ ladrillo & $56 \mathrm{~cm}$ & granito $(70 \mathrm{~cm})+$ ladrillo & $56 \mathrm{~cm}$ & Fábrica de ladrillo & $42 \mathrm{~cm}$ \\
\hline Sótano & Fábrica de ladrillo & $112 \mathrm{~cm}$ & Fábrica de ladrillo & $56 \mathrm{~cm}$ & Fábrica de ladrillo & $56 \mathrm{~cm}$ & Fábrica de ladrillo & $56 \mathrm{~cm}$ & Fábrica de ladrillo & $56 \mathrm{~cm}$ \\
\hline
\end{tabular}



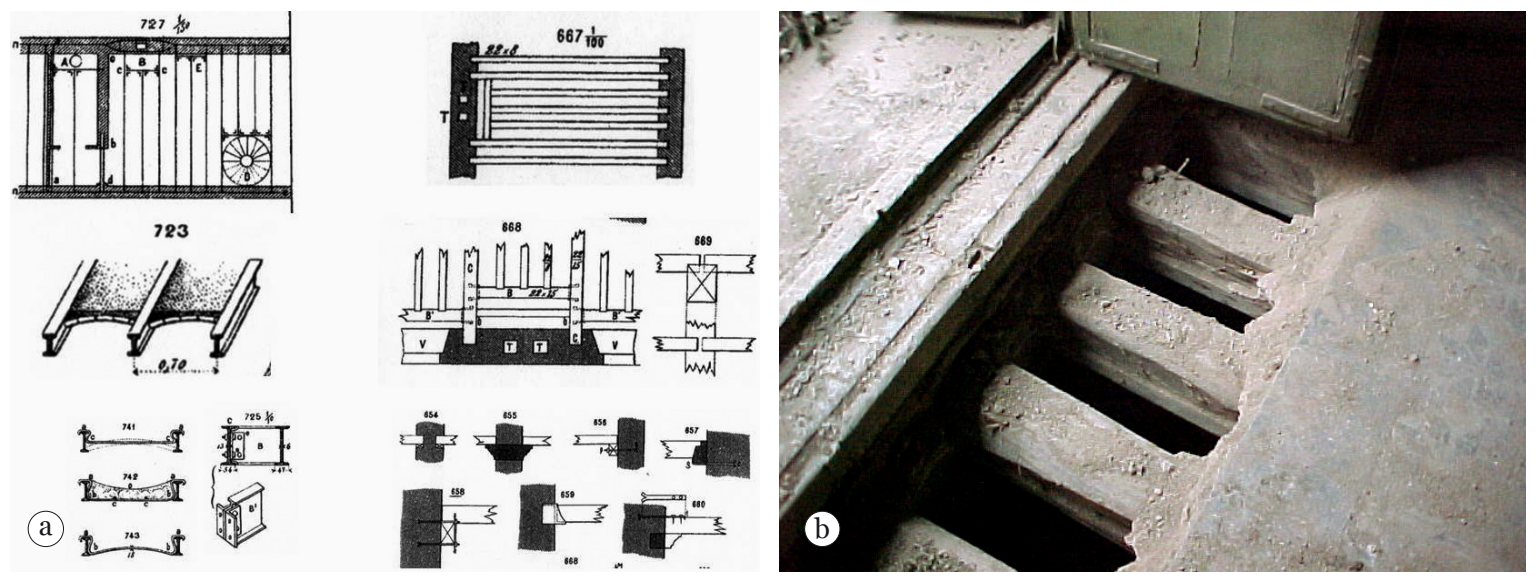

Figura 12. a) Forjados metálicos y forjados de madera (Ger y Lóbez 1898, Gaztelu 1899),

b) Forjados tradicionales de madera con solución de hueco por macizo en la c/Relatores.

pondientes también de madera. Los estribos son de sesma y los pares de escuadría proporcionada al ancho de los vanos que cubren según las dimensiones de cada faldón. Tienen un orden de zoquetes y van embarbillados a los estribos y picaderos. Se colocan gatillos de hierro para contrarrestar el empuje de los pares. El entablado de ripia se sienta con solape y va clavado con dos clavos en cada par. Sobre el entablado se coloca la teja de barro cocido a torta y lomo, a escantillón, con un tercio de entrega. Van guarneciendo los emboquillados, respaldos y caballetes y con escocias en los vuelos de medianerías. Las limas se cubren con plancha de plomo sobre cama de yeso, y tienen $35 \mathrm{~cm}$ de ancho por $10 \mathrm{~cm}$ de profundidad. El alero de las fachadas, es de canecillos dobles, con corona y tabicas de madera rodeada y labrada con entalladuras, y las coronas y chaperones del patio tienen una moldura. Los tratados de carpintería más singulares, entre otros, Gaztelu (29) recogen estos detalles constructivos.

\section{CONCLUSIÓN}

Se aborda el análisis constructivo de los edificios con entramado de madera, previo al proceso material de la rehabili- tación. El estudio de tres casas construidas en 1899, consideradas representativas de la transición de la construcción entramada y la introducción de los nuevos materiales: las columnas de fundición, las vigas metálicas y las escaleras de hierro, ha permitido avanzar algunas conclusiones. El proceso de sustitución de esta construcción entramada por fábrica de albañilería tiene sus comienzos a finales del s. XVIII tras el incendio de la Plaza Mayor de Madrid, en 1790. El punto de partida es el abandono de la construcción de las fachadas principales con entramado de madera y le sigue la sustitución de las medianerías entramadas por fábrica de ladrillo. La sustitución de los forjados de madera por forjados metálicos tiene su origen a finales del s. XIX con la introducción de las viguetas de acero en doble T aunque todavía conviven ambos tipos en un mismo edificio. El abandono de la madera en la construcción de las cubiertas es muy posterior y se continuará empleando en el s. XX. La comprensión global de estos sistemas permite recrear el proceso constructivo original y situar los daños más frecuentes en la mayor parte de los edificios construidos en Madrid antes de 1900 y que se han conservado.

\section{REFERENCIAS}

(1) Dutu, A., Gomes-Ferreiera, J., Guerreiro, L., Branco, F. Gonçalves, A.M. (2012). Timbered masonry for earthquake resistence in Europe. Materiales de construcción, 62(308): 615-628, doi: http://dx.doi.org/10.3989/mc.2012.01811.

(2) Ayuntamiento de Madrid. (2011). Nueva Ordenanza sobre Conservación, Rehabilitación y estado ruinoso de las edificaciones ANM 2011/65. Boletín Oficial del Ayuntamiento de Madrid, nº 6580.

(3) González, E., Aroca, R. (2001). Classification of masonry structures used in domestic architecture of 17th-19th centuries in Madrid. Structural Studies, Repairs and Maintenance of Historical Buildings, STREMAH. WITT PRESS.

(4) González, E., Aroca, R. (2003). Wooden framed structures in Madrid domestic architecture of the 17th to the 18th centuries. En First International Congress on Construction History, Madrid.

(5) Ayuntamiento de Madrid. (2010). Compendio de las Normas Urbanísticas del Plan General de Ordenación Urbana de Madrid PGOUM-97. Madrid: Área de Gobierno de Urbanismo y Vivienda.

(6) Langenbach, R. (2007). From "Opus Craticium" to the "Chicago frame": Earthquake-Resistant Traditional Construction. International Journal of Architectural Heritage, 1(1): 29-59, doi: http://dx.doi.org/10.1080/15583050601125998.

(7) Bell, P. (2009). The Structure of Georgian Houses. En Proceedings of the Third International Congress on Construction History, (pp. 167-174). Cottbus.

(8) Langenbach, R. (2008). Learning from the past to protect the future: Armature Crosswalls. Engineering Structures, 30(8): 2096-2100. doi: http://dx.doi.org/10.1016/j.engstruct.2008.04.028.

(9) Copani, P. (2007). Timber-frame building in Scandinavia: High Deformation Prevent the System from collapse. From material to Structure - Mechanical Behaviour and Failures of Timber Structures. En ICOMOS IWC - XVI International Symposium. Florence, Venice y Vicenza.

(10) Rejón-de Silva, A. (1788). Diccionario de las Nobles Artes para instrucción de los aficionados, y uso de los profesores. Imprenta de D. Antonio Espinosa. Ed. reimpresa Fundación Cultural COAM, 1995. 
(11) Langenbach, R. (2011). Ancient Construction Technologies that can protect modern building from collapse in earthquakes. En $4^{\text {th }}$ International Conference on Hazards and Modern Heritage. Sarajevo.

(12) Mascarenhas, J. (2005). Construction systems V. The pombalino buildings in Lisbon downtown. Lisboa: Ed. Livros Horizonte.

(13) Villanueva, J. (1790). Ordenanzas que se incluyen en el Informe enviado el 11 de octubre de 1790 al Ayuntamiento de Madrid. Madrid: A.S.A. 1-88-20.

(14) Gülkan, P., Langenbach, R. (2004). The earthquake resistence of traditional timber and masonry dwellings in Turkey. En 13th World Conference on Earthquake Engineering, paper $\mathrm{n}^{\circ} .2297$. Vancouver.

(15) Dogangun, A., Tuluk, I.O., Livoglu, R., Acar, R. (2006). Traditional wooden buildings and their damages during earthquakes in Turkey. Engineering Failure Anlysis, 13(6): 981-996, doi: http://dx.doi.org/10.1016/j.engfailanal.2005.04.011.

(16) Makarios, T., Demosthenous, M. (2006). Seismic response of traditional buildings of Lefkasm Island, Greece. Engineering Structures, 28(2): 264-278, doi: http://dx.doi.org/10.1016/j.engstruct.2005.08.002.

(17) Clairac-Sáenz, P. (1877). Diccionario General de Arquitectura e Ingeniería. Madrid: Imprenta Zaragozano y Jaime.

(18) Ger-Lóbez, F. (1898). Manual de Construcción Civil. Badajoz: La Minerva Extremeña.

(19) Castro, C. M. (1860). El Ensanche de Madrid o Plan Castro. Mapa del Proyecto del Ensanche y la Villa de Madrid.

(20) COAM. (1991). Guía de Arquitectura y Urbanismo de Madrid. Tomo II: Ensanche y Crecimiento (3 ${ }^{\mathrm{a}}$ Ed). Madrid: Colegio Oficial de Arquitectos.

(21) González, E. (2012). Structure of Houses in Madrid: Buildings and Documents (1669-1900). En 4th International Congress on Construction History. Paris.

(22) Bails, B. (1802). Diccionario de Arquitectura civil. Madrid: Imprenta de la viuda de Ibarra.

(23) García-Gómez, F. (1854). Revista de Obras Públicas, 17: 209-211.

(24) Marcos-Bausá, R. (1879). Manual del Albañil ( $3^{\mathrm{a}}$ ed). Madrid: Dirección y Administración.

(25) Gaceta de obras públicas. (1892). Ordenanzas Municipales de Madrid. Madrid: Imprenta de J. Perales-Martínez

(26) AHPM. Contrato para el derribo de una casa y construcción de tres, en la c/ del Pez con vuelta a la c/ de las Pozas y c/ de las Minas, (1899). AHPM n⿳ 38.028, fol. 188-196.

(27) San Nicolás, F. L. (1639). Arte y Uso de Arquitectura. Primera Parte. (Ed. Facs. 1989). Valencia: Albatros Ed.

(28) Torija, J. (1760). Tratado breve sobre las Ordenanzas de la Villa de Madrid, y policía de ella. Madrid: A. Pérez-de Soto impresor.

(29) Gaztelu, L. (1899). Carpintería de Armar. En Barré, L. A. (Ed.), Pequeña Enciclopedia Práctica de la Construcción, nº 4. Madrid: Librería editorial de Bailly-Bailliere e hijos. 